\title{
Implantation immédiate après extraction dentaire. A propos de trois cas cliniques colligés à Dakar
}

\author{
Immediate implantation after dental extraction: about 3 cases collected in Dakar
}

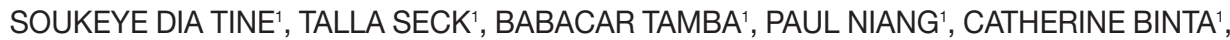 \\ BARRY GASSAMA ${ }^{1}$, AWA DIOUF ${ }^{1}$, YEHYIA FARHAT ${ }^{2}$, BOUBACAR DIALLO'
}

\begin{abstract}
RÉSUMÉ
La technique standard de pose d'implant dentaire préconise de différer l'acte de quelques mois par rapport à l'extraction dentaire. Outre l'allongement excessif de la durée du traitement, cette attente favorise la résorption osseuse. Par contre la mise en place immédiate d'un implant après extraction dentaire permet de limiter la perte osseuse, de préserver l'esthétique par la conservation de la table osseuse vestibulaire, de profiter de l'intense vascularisation créée par l'extraction et de réduire le temps du traitement.

L'objectif de ce travail est de rapporter trois cas cliniques d'implantation immédiate traités à Dakar et d"étudier les aspects cliniques et les modalités techniques de l'implantation immédiate dans notre pratique. Les indications d'extraction dentaire ont été : un faux canal, une fracture corono-radiculaire, et une fracture radiculaire. Les implants ont été posés au maxillaire (incisive latérale et $2^{\mathrm{e}}$ prémolaire).

Le bilan radiographie a comporté pour chaque cas un orthopantomogramme et des radiographies rétro-alvéolaires. Des implants cylindro-coniques et vissés du système TBR ont été utilisés. Leurs longueurs étaient supérieures à $13 \mathrm{~mm}$ avec des diamètres de 3,5 et $4 \mathrm{~mm}$. En effet il fallait tenir compte du diamètre de la racine extraite, de sa longueur et de sa rétention dans la région apicale.

Les membranes en titane (Biotane ${ }^{\circledR}$ ) non résorbables ont été utilisées pour le traitement des défauts osseux péri-implantaires, favorisant ainsi une bonne régénération. Après deux ans de mise en fonction le résultat fonctionnel et esthétique était satisfaisant.

La demande en thérapeutique implantaire est toujours limitée au Sénégal car le coût du traitement reste encore élevé. Promouvoir l'implantologie en pratique hospitalo-universitaire permettrait une meilleure accessibilité aux patients de ce type de traitement. (Med Buccale Chir Buccale 2009 ; 15: 199-208).

mots clés: extraction dentaire, implantation immédiate, régénération osseuse guidée, ostéointégration, implant dentaire
\end{abstract}

\section{SUMMARY}

The standard technique of dental implantation recommend to differ the act a few months after tooth extraction. In addition to excessive lengthening of the duration of treatment, this expectation promotes bone loss. As against the immediate implantation after tooth extraction makes possible to limit bone loss, to preserve aesthetics by the retention of bone vestibular table, to benefit of the intense vascularization created by the extraction and to reduce time of treatment.

The aim of our study was to report three cases of immediate implantation realised in Dakar and to describe the clinical and technical aspects for the immediate implantation in our practice. The causes of tooth extraction was: a false channel, a corono-root fracture and a root fracture. The implants were placed in the maxilla, (lateral incisor

\footnotetext{
1. Service de Chirurgie buccale Département d'Odontologie Université Cheikh Anta Diop Dakar
}

2. Praticien privé Dakar

Demande de tirés à part:

Soukèye Dia Tine Service de Chirurgie buccale Département d'Odontologie Université Cheikh Anta Diop BP 5005 Dakar Fann diatine@ hotmail.com 
and second premolar). The cylindrical-conical implant and screwed TBR system was used. Their lengths were greater than $13 \mathrm{~mm}$ with diameters of 3.5 and $4 \mathrm{~mm}$. Indeed it should be given to the diameter of the root extract, its length and its retention in the apical level. Titanium membranes (Biotane $\left.{ }^{\circledR}\right)$ were used for the treatment of bone defects around of implant, to promote a good regeneration of the bone. Two years after treatment, the functional and aesthetic result was satisfactory

The demand for therapeutic implantation is still weak because, the treatment cost remains very high. Its development in practice teaching hospital would allow a better accessibility to the patients of this type of treatment. (Med Buccale Chir Buccale 2009 ; 15: 199-208).

key words: dental extraction, immediate implantation, guided bone regeneration, osseointegration, dental implant

médecine

buccale chirurgie buccale

VOL. $15, \mathrm{~N}^{\circ} 4$ 2009

page 200
L'implantation immédiate consiste à placer un implant dans l'alvéole vide, aussitôt après l'extraction dentaire. Ce concept diffère de celui de Branemark qui préconise une chirurgie implantaire sur un os parfaitement cicatrisé ${ }^{[2]}$. En effet, cette technique classique nécessite un temps d'attente de neuf mois après une extraction avant de mettre en place un implant. Outre l'allongement excessif de la durée du traitement, cette attente favorise la résorption osseuse, pouvant rendre impossible la mise en place de l'implant. Par contre l'implantation immédiate permet de sauvegarder l'os alvéolaire en hauteur et en épaisseur vestibulo-linguale. Elle permet d'éviter la résorption complète des procès alvéolaires par les phénomènes inflammatoires.

L'objectif de ce travail est de rapporter trois cas cliniques d'implantation immédiate traités à Dakar et d'étudier les aspects cliniques et les modalités techniques de l'implantation immédiate dans notre pratique.

On a sélectionné trois cas cliniques colligés en 2006 dans le cadre de la pratique à Dakar. Chaque dossier de patient comportait : un examen clinique, un bilan radiologique et un protocole opératoire.

\section{CAS CLINIQUE $\mathbf{N}^{\circ} \mathbf{1}$}

Monsieur D. F. est un ingénieur âgé de 49 ans. II a consulté pour une douleur chronique sur la 12. L'examen radiologique a révélé un faux canal sur la dent (Fig. 1). L'interrogatoire n'ayant décelé aucune pathologie, l'indication d'extraction suivie de la mise en place immédiate d'un implant a été posée.

Une empreinte des maxillaires à l'alginate a été réalisée pour la confection d'une prothèse immédiate provisoire amovible. Une prescription comportant un antibiotique (amoxicilline 2g/j) pendant 8 jours, un antiinflammatoire et un bain de bouche a été donnée. L'extraction atraumatique de la dent a été réalisée sous anesthésie locale para-apicale, suivie immédiatement de la mise en place d'un implant de type TBR autotaraudant, vissé, avec une longueur de 15,5 mm et un diamètre de $3,5 \mathrm{~mm}$ (Fig. 1.2 à 1.5).

Aucun matériau de comblement n'a été utilisé. Cependant, une membrane en titane (Biotane ${ }^{\circledR}$ ) a été posée et maintenue par la vis de fermeture de l'implant (Fig. 1.6). Puis le site opératoire a été recouvert par le lambeau en assurant le repositionnement coronaire avec un point de suture en surjet. La prothèse provisoire a été mise en place une semaine plus tard et une radiographie de contrôle effectuée (Fig. 1.7). La membrane a été déposée 3 mois après et le pilier transgingival mis en place (Fig. 1.8). Un mois après la cicatrisation, le faux moignon en titane a été posé et taillé à $15^{\circ}$ à l'aide de fraise en acier tungstène (Fig. 1.9). Deux mois après la taille du faux moignon, l'empreinte a été prise, puis la prothèse scellée une semaine plus tard (Fig. 1.10). Le patient a été suivi régulièrement. Un an après, une radiographie panoramique et une radiographie rétro-alvéolaire ont montré une bonne intégration osseuse de l'implant. Le contrôle à deux ans a été satisfaisant sur le plan fonctionnel et esthétique. 


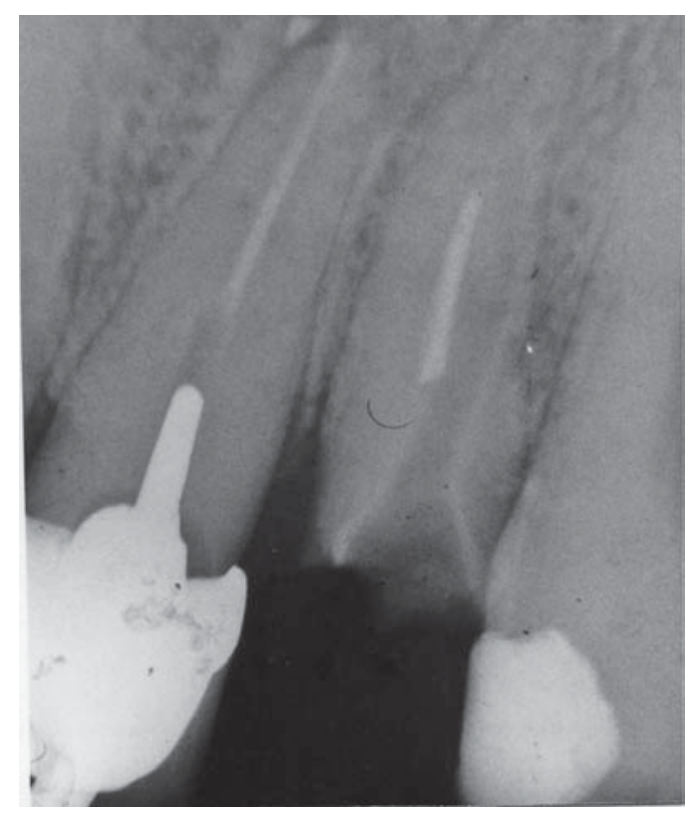

Figure 1.1: Radiographie pré-opératoire montrant un faux canal sur la 22.

Preoperative radiography showing a false canal on teeth 22.

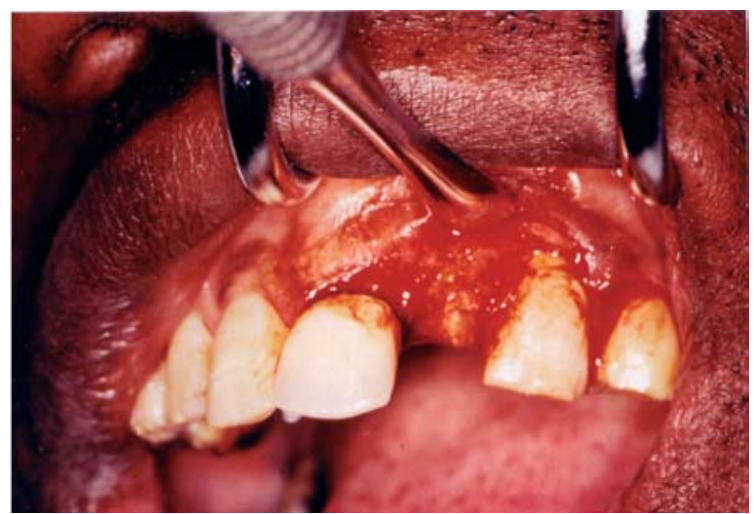

Figure 1.2 : Avulsion atraumatique de la 22. No traumatic avulsion of teeth 22.

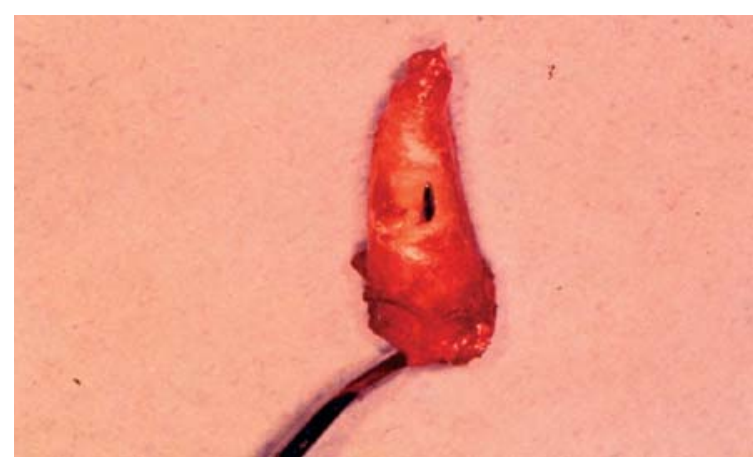

Figure 1.3: Sonde montrant le faux canal après extraction.

Probe showing the false canal after extraction.

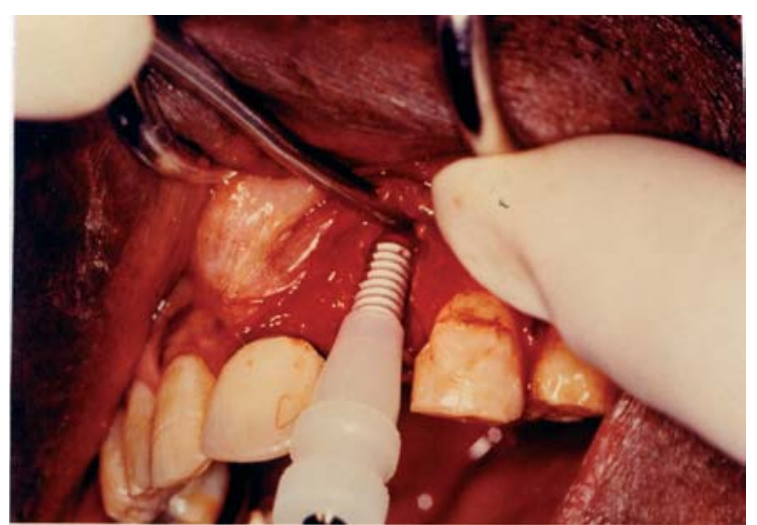

Figure 1.4 : Mise en place l'implant autotaraudant. Implant in place.

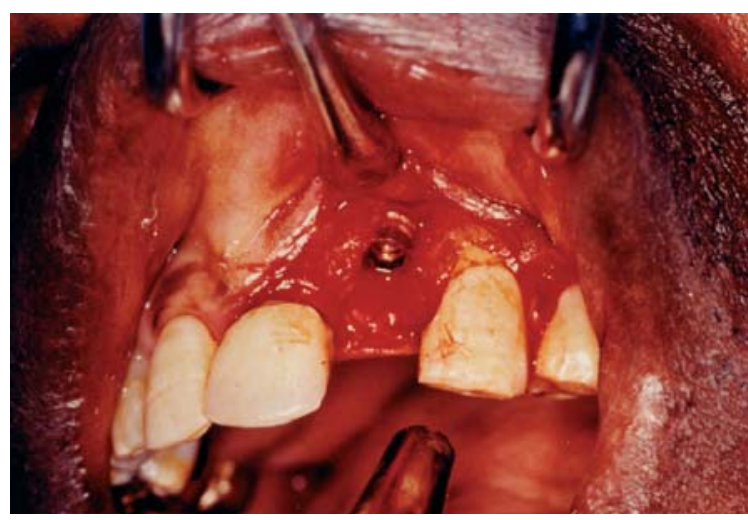

médecine buccale chirurgie buccale VOL. $15, \mathrm{~N}^{\circ} 4$ 2009 page 201

Figure 1.5 : Implant en place. Implant in place.

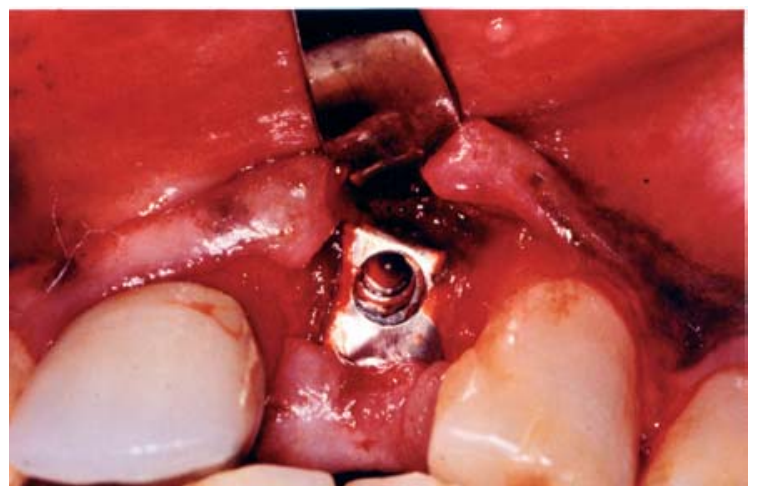

Figure 1.6 : Membrane mise en place. Implementation of the membrane. 


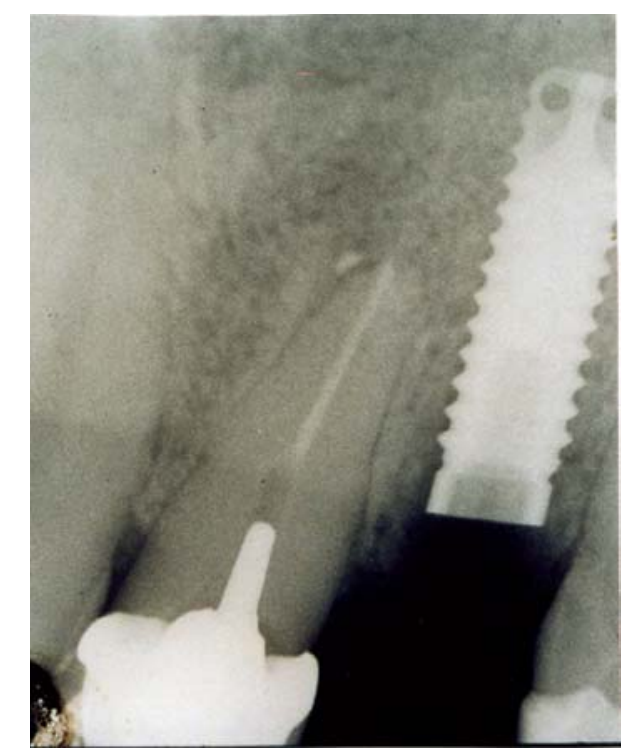

Figure 1.7 : Radiographie de contrôle après 3 semaines. Control radiography three weeks later.

médecine

buccale chirurgie buccale

VOL. $15, \mathrm{~N}^{\circ} 4$ 2009

page 202

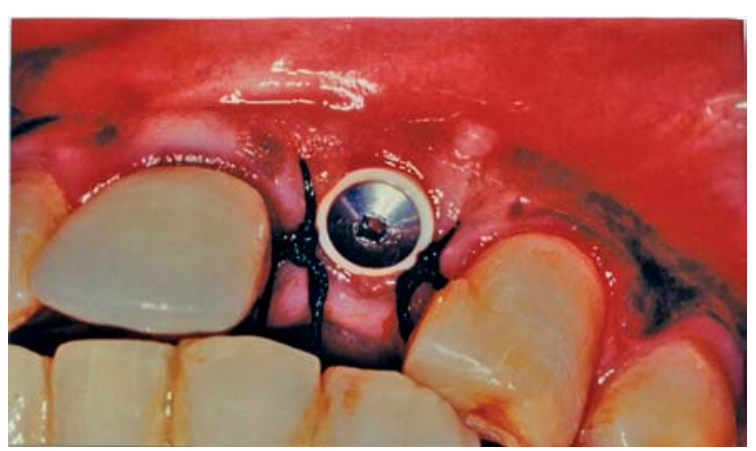

Figure 1.8: Mise en place du pilier trans-gingival en zircone 6 mois plus tard.

Zircon transgingival pillar in place six months later.

\section{CAS CLINIQUE $\mathbf{N}^{\circ} 2$}

Madame H. B., âgée de 46 ans, consulte pour une fracture corono-radiculaire de la 14 qui avait subi une biopulpectomie un an auparavant (Fig. 2.1). L'indication d'extraction suivie d'une implantation immédiate a été posée. L'examen pré-implantaire classique a été fait, des mesures prophylactiques et un contrôle de plaque ont permis de maintenir une gencive péri-dentaire exempte d'inflammation. Le bilan médical général n'a décelé aucune pathologie contre-indiquant la pose d'un implant.

La technique chirurgicale a été la même que précédemment. Un implant de type TBR autotarodant,

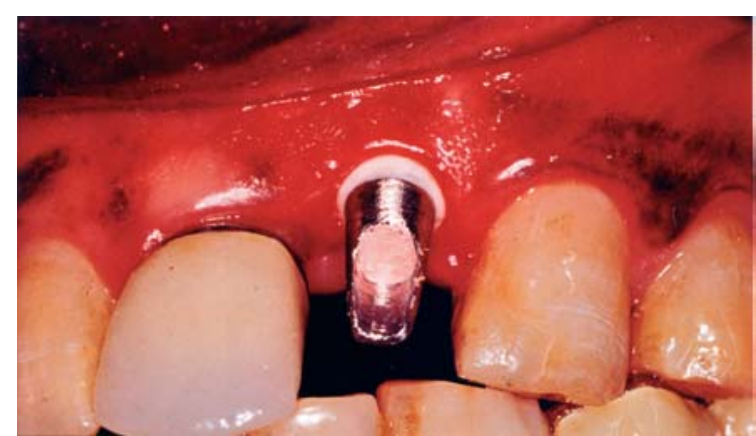

Figure 1.9 : Faux moignon vissé sur le pilier transgingival.

False strub screwed on the transgingival pillar.

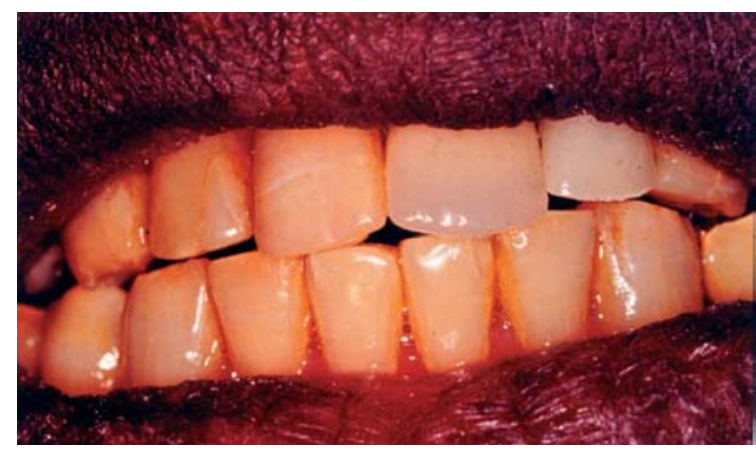

Figure 1.10 : Prothèse définitive scellée. Sealed definitive prosthesis.

vissé, de $13 \mathrm{~mm}$ de longueur et $4 \mathrm{~mm}$ de diamètre a été posé. Le site opératoire a été suturé avec un surjet simple (Fig. 2.2).

II y a eu une exposition de la membrane au $20^{\circ}$ jour ; quelques points de suture ont été refaits pour la maintenir si possible enfouie. Deux mois plus tard, la membrane a été déposée et le pilier transgingival mis en place. Puis le lambeau a été repositionné coronairement et suturé (Fig. 2.3). Un mois après la cicatrisation, une empreinte a été prise pour la réalisation de l'armature métallique. Une semaine après l'essayage de l'armature, la pose de la couronne céramo-métallique (Fig. 2.4) avec scellement provisoire a été effectuée. Elle a été suivie du scellement définitif la semaine suivante.

Les résultats après deux ans de mise en fonction ont montré une absence de mobilité, de douleur et d'image péri-implantaire sur la radiographie rétroalvéolaire. 


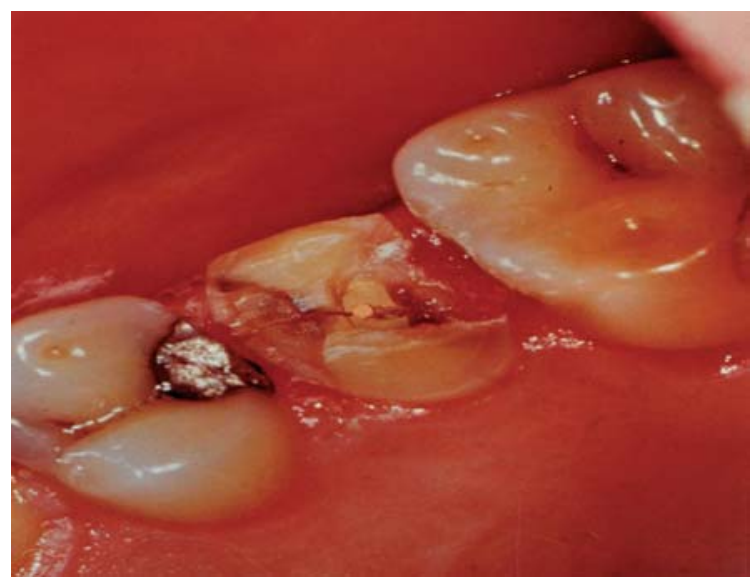

Figure 2.1 : Fracture coronoradiculaire de la 15. Coronoradicular fracture of tooth 15.

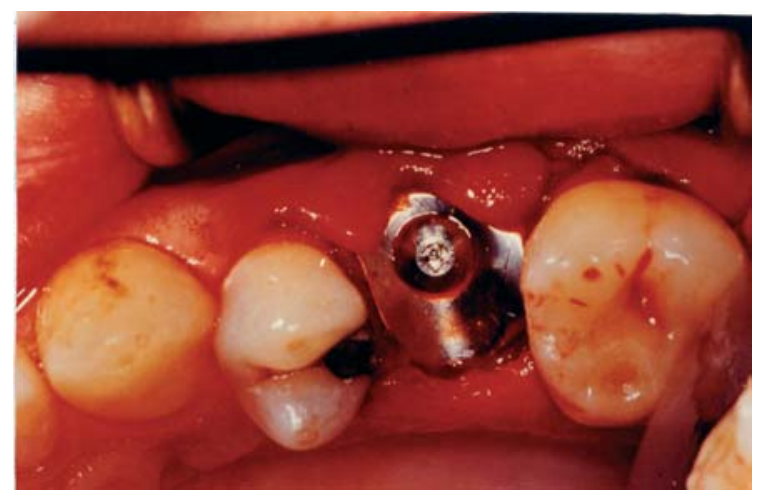

Figure 2.2: Implant et membrane en place. Implant and membrane in place.

\section{CAS CLINIQUE N³}

Monsieur T. G., étudiant de 19 ans, a consulté pour une douleur sur la 22. II avait subi un traumatisme maxillo-facial lors d'un accident de la voie publique un an auparavant. L'examen clinique a décelé une absence de vitalité sur les trois incisives supérieures $(11,21$ et 22), associée à une dyschromie. L'examen radiographique a montré un traitement endodontique sur les trois dents avec obturation des canaux et un trait de fracture radiculaire au 1/3 apical sur la 22 (Fig. 3.1). L'indication d'extraction et la mise en place immédiate d'un implant a été posée. Une prescription d'antibiotique, d'anti-inflammatoire et de bains de bouche a été établie. La technique chirurgicale a été la même que précédemment (Fig. 3.2). Un implant TBR autotarodant,

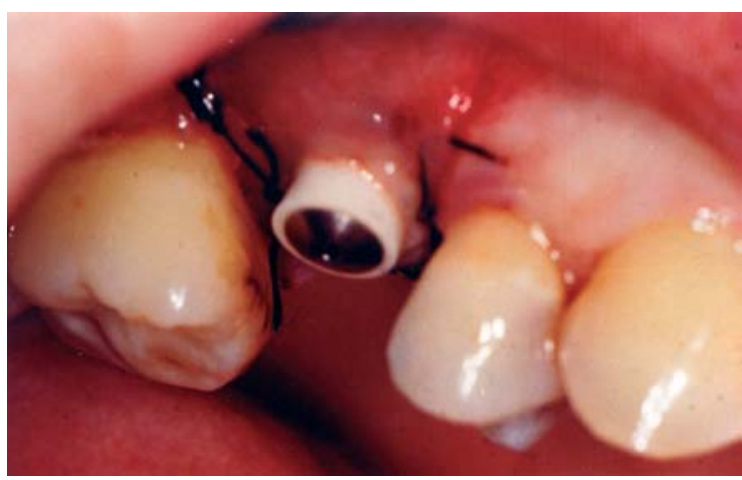

Figure 2.3: Mise en place du pilier transgingival et suture.

Transgingival pillar in place and suture.

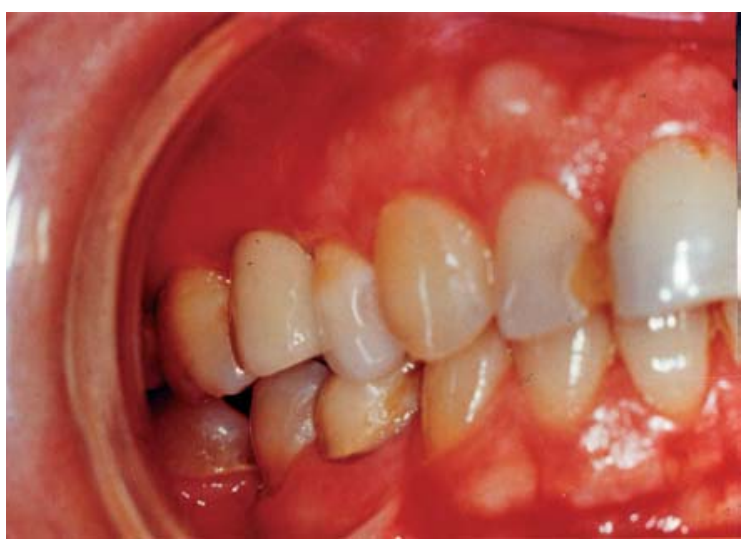

médecine buccale chirurgie buccale VOL. $15, \mathrm{~N}^{\circ} 4$ 2009 page 203
Figure 2.4 : Prothèse définitive scellée. Sealed definitive prosthesis.

vissé, d'une longueur de 15,5 mm et un diamètre de 4 mm (Fig. 3.3) a été utilisé. Une membrane en titane a été ajustée et les lambeaux suturés (Fig. 3.4). Un examen de contrôle a été effectué trois jours plus tard. Un bridge provisoire a été réalisé de la 11 à la 22. Après trois mois de cicatrisation, la membrane en titane a été déposée et la vis de cicatrisation posée dans le même temps. Le faux moignon a été mis en place une semaine plus tard et la taille effectuée. La prothèse provisoire a été rebasée et l'empreinte réalisée. L'essayage des armatures métalliques a été effectué une semaine après la prise d'empreinte : la 11 et la 21 étant solidarisées, la 22 isolée. La pose des prothèses définitives a été faite la semaine suivante (Fig. 3.6). Un suivi régulier pendant 2 ans a permis de contrôler et d'apprécier la bonne ostéo-intégration (Fig. 5). 


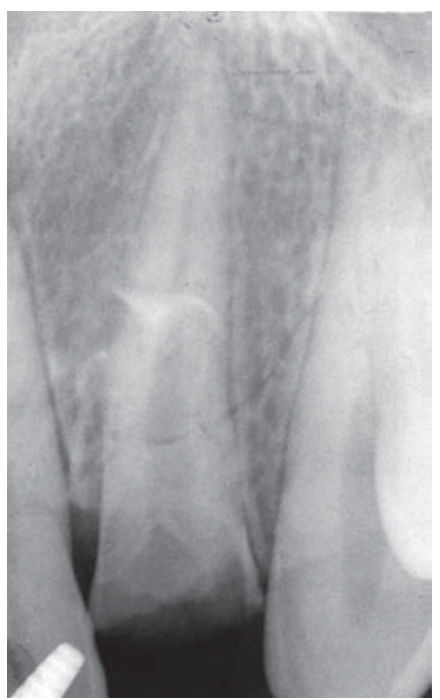

Figure 3.1 : Radiographie préopératoire : fracture radiculaire horizontale de la 22.

Preoperative radiography: horizontal root fracture of tooth 22.

médecine

buccale

chirurgie

buccale

VOL. $15, \mathrm{~N}^{\circ} 4$ 2009

page 204

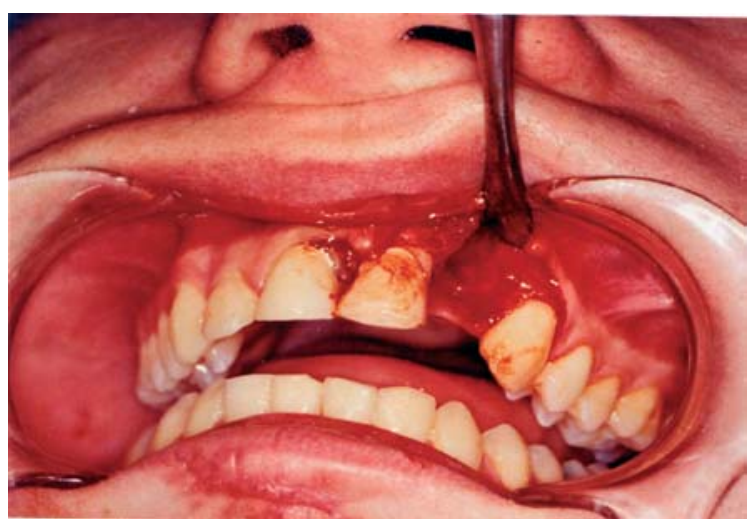

Figure 3.2 : Avulsion de la 22.

Avulsion of tooth 22.

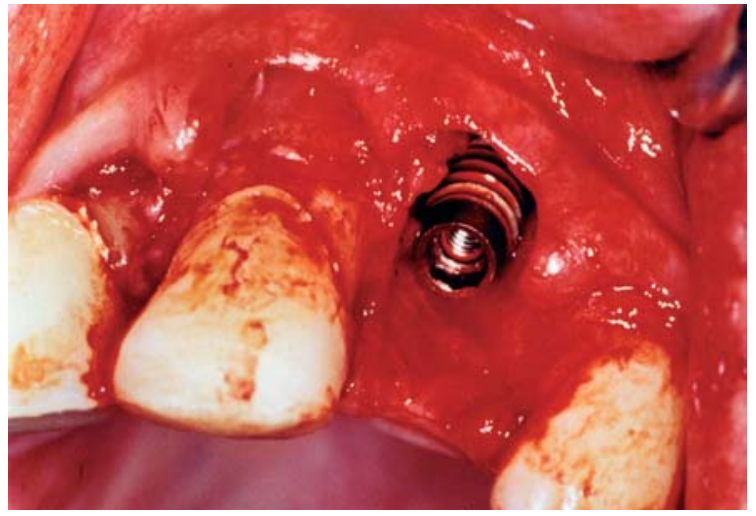

Figure 3.3 : Mise en place de l'implant. Implant in place.

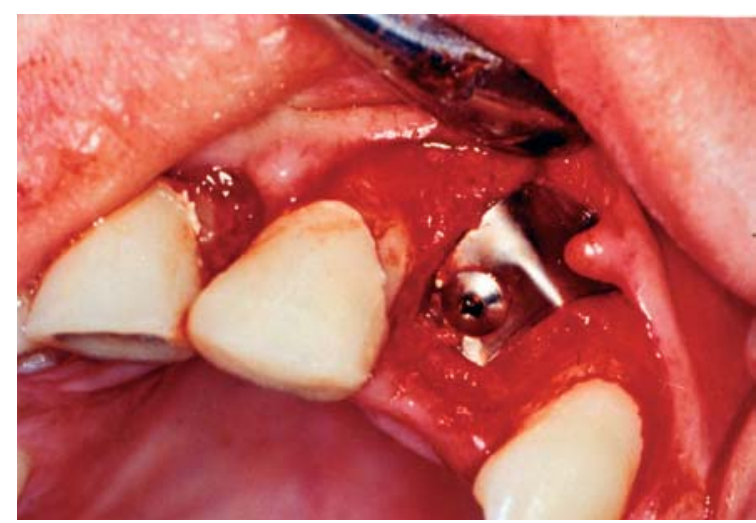

Figure 3.4 : Membrane en place retenue par la vis de couverture.

Membrane in place booked by the cover screw.

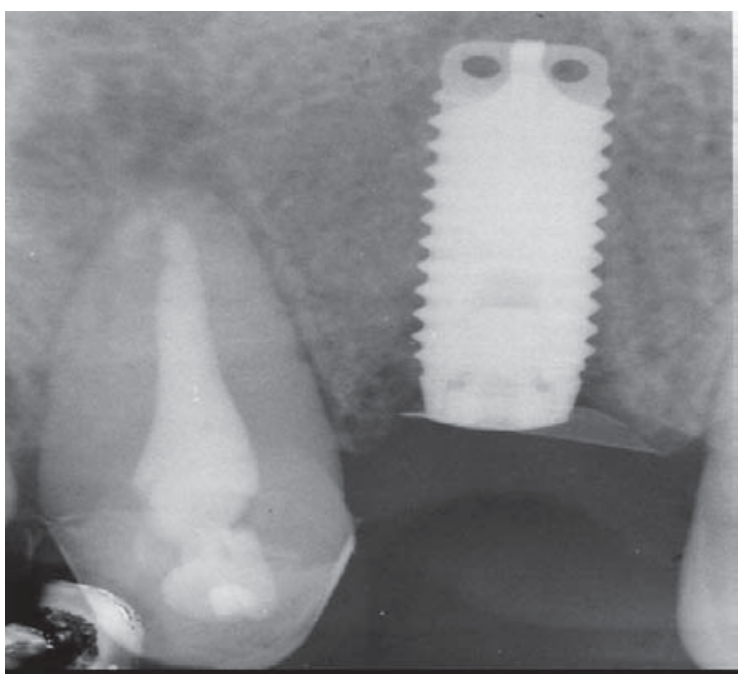

Figure 3.5 : Radiographie de contrôle 6 mois plus tard. Control radiography six weeks later.

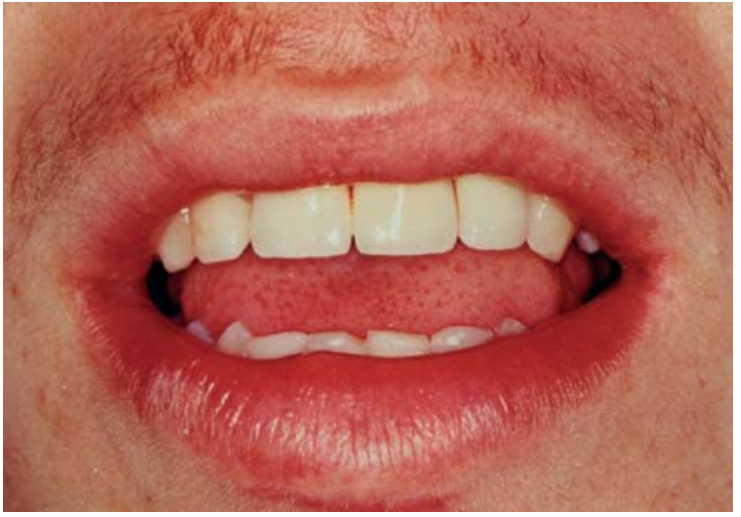

Figure 3.6 : Prothèse définitive scellée. Sealed definitive prosthesis. 


\section{DISCUSSION}

II existe de nombreuses indications pour les extractions dentaires : les caries, les parodontopathies, les rhizalyses, les échecs endodontiques, les traumatismes, les indications d'ordre prothétique, l'orthodontie. D'après plusieurs études la carie demeure la principale cause d'extraction suivie par les parodontopathies ; par contre, il est noté une faible prévalence d'extraction après traumatisme ${ }^{[4,9] .}$

Dans notre étude, les causes d'extraction dentaire ont été : un cas de faux canal, une fracture corono-radiculaire, une fracture radiculaire. La fracture radiculaire est secondaire à un accident de la voie publique. Le faux canal et la fracture corono-radiculaire étaient d'origine iatrogène par un tenon radiculaire trop important.

Dans une étude réalisée par Krennmair et coll. [12] en Australie, sur 112 patients candidats à la pose d'implants, 67 étaient des femmes. Dans les travaux de Fugazzotto ${ }^{[10]}$, sur 57 patients réhabilités par implants, 36 étaient des femmes. Ces études confirment l'intérêt que portent les femmes à la thérapeutique implantaire. Cependant, dans notre étude, parmi les trois cas cliniques sélectionnés, les deux concernaient des hommes.

D'une manière générale, les patients candidats à la pose d'implant sont relativement âgés (en moyenne 51,4 ans) [8]. L'âge avancé n'est pas une contre-indication absolue à la pose d'implants dentaires. Cependant, il convient de s'assurer de l'état général du patient, de sa motivation et de son aptitude mentale à recevoir des implants. Chez l'enfant ou l'adolescent, les différentes études montrent que l'implant se comporte comme une dent ankylosée et ne suit pas la croissance verticale des maxillaires [11]. II est donc impératif d'attendre la fin de croissance des maxillaires avant d'envisager une thérapeutique implantaire chez l'adolescent. Dans notre étude, on sélectionne un sujet jeune (19 ans) dont l'indication d'extraction a été posée suite à un traumatisme; les deux autres patients sont âgés de 46 et 49 ans.

Il est classique de dire que tout site d'extraction d'une dent mono-radiculée est implantable immédiatement ${ }^{[6]}$; c'est le cas des secteurs incisivo- canins supérieur et inférieur ainsi que de la première prémolaire inférieure et des prémolaires supérieures. Pour ce qui est de la deuxième prémolaire inférieure, le risque majeur est l'émergence du trou mentonnier. Il en est de même pour les molaires inférieures avec le canal dentaire inférieur. Or le principe d'une implantation immédiate est de forer au-delà de la limite apicale de la dent de façon à conférer à l'implant une bonne stabilité primaire lors de la période de cicatrisation. Même pour les molaires supérieures, Lazzara [13] a obtenu des résultats favorables avec l'implantation immédiate.

En implantologie dentaire, l'imagerie pré-opératoire est un examen indispensable pour la connaissance anatomique du site osseux et de ses caractéristiques. Une première approche est faite par des examens radiologiques classiques tels que les radiographies panoramique et rétroalvéolaire, puis elles seront complétées par des examens plus précis (scanner, scanora ou dentascan), qui permettent d'obtenir des informations beaucoup plus précises sur le site osseux implantaire [14]. Ces techniques modernes sont très onéreuses et ne sont pas toujours disponibles surtout dans les pays aux ressources limitées comme le Sénégal, où il existe cependant des besoins en implantologie dentaire ${ }^{[8]}$.

Dans notre étude, les implants ont été posés en se basant sur des examens radiographiques de types standard : la radiographie panoramique et les radiographies rétro-alvéolaires. Le cliché panoramique présente un intérêt pour établir rapidement un état sommaire et général de la denture et pour dépister des lésions importantes. II apporte malgré ses imperfections, une grande quantité d'informations sur un seul cliché. La radiographie rétro-alvéolaire est un examen effectué au cabinet dentaire et son coût est très faible. Elle donne d'excellents résultats sur la qualité et la hauteur d'os disponible dans la région du site implantaire. Ces deux incidences combinées à l'examen local donnent souvent les renseignements nécessaires en secteur antérieur pour la pose d'implants immédiatement après extraction ${ }^{88}$. Cependant, dans certains cas, l'examen tomographique est indispensable pour analyser le site osseux en zone antérieure. médecine buccale chirurgie buccale VOL. $15, N^{\circ} 4$ 2009 page 205 
médecine
En implantologie immédiate, les implants les plus utilisés actuellement sont de forme cylindrique ou cylindro-conique et vissés. Leur fonctionnalité sur des décennies, sans complications cliniques, a été démontrée [7]. La présence de pas de vis augmente la surface de contact entre l'implant et l'os, améliore la stabilité initiale, la résistance aux forces de cisaillement et la distribution des forces dans le tissu osseux. Dans notre étude, le système TBR cylindro-conique, vissé, a été utilisé pour tous les cas cliniques. Ces implants ont été choisis pour les caractéristiques suivantes :

- un octogone interne permettant un blocage antirotationnel du moignon,

- un col lisse sur 1,5 mm de hauteur, favorisant une bonne hygiène,

- une base cruciforme assurant un blocage antirotationnel de l'implant,

- une bonne ergonomie d'utilisation avec une manipulation relativement facile.

Ce type d'implant a été mis au point par Benhamou ${ }^{[3]}$ et présente une fiabilité à long terme de plus de 15 ans.

Selon Davarpanah et coll. [7] les implants courts de $7 \mathrm{~mm}$ et $8,5 \mathrm{~mm}$ de longueur ont des taux de succès inférieurs à ceux dont la longueur est supérieure à $10 \mathrm{~mm}$. Dans notre étude, les implants utilisés ont des longueurs supérieures ou égales à $13 \mathrm{~mm}: 15,5 \mathrm{~mm}$ pour les incisives latérales avec un diamètre de 3,5 ou de $4 \mathrm{~mm}$, et $13 \mathrm{~mm}$ pour la prémolaire avec un diamètre de $4 \mathrm{~mm}$. En effet il faut tenir compte non seulement du diamètre de la racine extraite mais également de sa longueur et de la rétention dans la région apicale. Selon l'OMS, lorsque le type et la longueur de l'implant choisi sont bien adaptés au site osseux, le taux de succès est de $98 \%$ à la mandibule et $97 \%$ au maxillaire pour 2000 implants posés avec un recul de 8 ans.

L'utilisation d'une membrane est indiquée dans les cas de déficit de tissu osseux sur le site implantaire, entraînant une déhiscence entre l'implant et le rebord osseux. Actuellement, leur utilisation est contre-indiquée en l'absence de défaut osseux. Dans notre étude, les membranes en titane $\left(\right.$ Biotane $\left.^{\circledR}\right)$ non résorbables ont été utilisées pour le traitement des défauts osseux péri-implantaires, favorisant ainsi une régénération satisfaisante. De nombreux auteurs comme Lazzara [13], puis Caudill Meffert ${ }^{[5]}$ ont confirmé la meilleure efficacité des membranes non résorbables dans la régénération osseuse guidée péri-implantaire. La technique opératoire standard oblige à attendre neuf mois après une extraction avant de mettre en place un implant. Outre l'allongement excessif de la durée du traitement, cette attente favorise la résorption osseuse, pouvant rendre impossible la mise en place de l'implant ou créer des problèmes esthétiques difficilement surmontables. La mise en place immédiate permet de limiter la perte osseuse, de préserver l'esthétique par la conservation de la table osseuse vestibulaire, de profiter de l'intense vascularisation créée par l'extraction et de réduire le temps du traitement.

Il est important de disposer d'un volume osseux suffisant à l'apex de l'implant, permettant de bien l'immobiliser dans un os sain. II faut en outre disposer de suffisamment de tissu gingival pour recouvrir le site d'extraction. Ce tissu est trouvé en tractant la gencive en direction coronaire. Les espaces entre l'alvéole et l'implant doivent être obturés par un matériau de comblement ou recouverts par une membrane.

Les résultats de l'ostéo-intégration en implantologie immédiate sont semblables ou même meilleurs que ceux obtenus avec des implants mis en place dans des sites cicatrisés. Schavartz-Arad et coll. [17] ont posé sur 43 patients, 117 implants immédiats et 263 implants à pose différée. Après un suivi de 5 ans, ils ont obtenu $96 \%$ de succès pour les implants immédiats et $89,4 \%$ de succès pour les implants à pose différée.

Aires ${ }^{[1]}$ et coll. ont mis en place sur 7 patients, 29 implants immédiats et 33 implants à pose différée. lls ont obtenu sur une période de 4 mois $96,6 \%$ de succès pour les implants immédiats et $96,5 \%$ de succès pour les implants à pose différée.

Nemcovsky et coll. [15] ont fait deux études différentes. D'abord sur 24 patients où 31 implants ont été posés immédiatement après extraction. Ils ont obtenu $97,2 \%$ de succès avec un temps de suivi de 6 à 8 mois. Puis sur 19 patients avec 23 implants placés tardivement et ont obtenu sur la même période $90,2 \%$ de succès.

La technique d'implantation post-extractionnelle semble donner un meilleur résultat, avec une cica- 
trisation osseuse plus rapide qui pourrait s'expliquer par la vascularisation liée à la présence du desmodonte, par l'ouverture des espaces médullaires et le moindre échauffement dû au forage [19]. Cependant il existe des inconvénients de deux ordres:

- la difficulté de recouvrir complètement le site implantaire avec les tissus mous,

- la stabilité primaire de l'implant parfois insuffisante.

Toutefois, les avantages décrits et les résultats positifs obtenus plaident en faveur de cette technique.

Actuellement, les résultats semblent optimisés par la présence de membranes de cicatrisation recouvrant le site implantaire, avec ou sans greffe de comblement. La mise en place immédiate d'un implant après extraction, associée à l'utilisation de matériaux de comblement et de membranes, constitue une technique chirurgicale de pointe en chirurgie reconstructrice pour obtenir un environnement osseux favorable autour d'un implant stable avec un excellent pronostic ${ }^{[16,18]}$. Les membranes utilisées ont été bien tolérées par les patients et ont été laissées en place jusqu'à la deuxième phase chirurgicale, évitant ainsi une intervention supplémentaire. C'est donc une technique particulièrement intéressante qui a permis de préserver au maximum le capital osseux de départ et de diminuer la durée du traitement.
Cependant, quelques difficultés ont été rencontrées concernant le deuxième cas clinique, avec l'exposition de la membrane au $20^{\circ}$ jour après sa pose. Quelques points de sutures ont été refaits, l'antibiothérapie prolongée d'une semaine, complétée par des bains de bouche et l'application d'un gel de chlorhexidine à 0,2 \% trois fois par jour sur la zone suturée. Après une semaine, on a constaté une parfaite fermeture de la gencive.

\section{CONCLUSION}

La mise en place d'un implant endo-osseux dans un alvéole immédiatement après l'extraction d'une dent, représente une option thérapeutique intéressante, lorsque l'indication est bien posée. C'est une technique qui permet d'éviter la résorption osseuse post-extractionnelle et autorise ainsi l'utilisation des implants dans des zones où l'évolution de la crête alvéolaire après extraction aboutirait à un volume osseux insuffisant.

L'implantation immédiate permet aussi de combiner la cicatrisation post-extractionnelle avec la période nécessaire à l'obtention de l'ostéointégration. Ainsi le nombre d'intervention et la durée du traitement sont réduits. Et le protocole chirurgical est plus simple que la technique de pose d'implants standard.

\section{RÉFÉRENCES}

1 - Aires I, Berger J. Immediate placement inextraction sites followed by immediate loading: a pilot study and case presentation. Implant Dent 2002 ; 11 : 87-94.

2 - Arquie H. Brefs rappels historiques sur l'implantologie en général, et les implants aiguilles en particulier. Rev Odonto-Implantol $1968 ; 17: 16$.

3 - Benhamou A. Biointégration en implantologie orale : implant TBR. Chir Dent Fr $1990 ; 22$ : 37-46.

4 - Cahen PM, Frank RM, Turlot JC. A survey of the reasons for dental extractions in France. J Dent Res 1985 ; 64 : 1087-93.

5 - Caudill RF, Meffert RM. Analyse histologique de l'ostéointégration d'implants endo-osseux placés dans des alvéoles artificiellement créées, avec et sans barrière de PTFE-e : 1ere partie : résultats préliminaires. Rev Int Parodont Res1991; $11:$ 207-15.
6 - Champagnat JF. L'implantologie immédiate, contre-indications, méthodes chirurgicales. Thèse Chirurgie dentaire $2^{\circ}$ cycle, Reims, 1992.

7 - Davarpanah M, Martinez H, Kebir M, Tecucianu JF. Manuel d'implantologie clinique. CdP, Paris, 2000.

8 - Dia Tine S. Analyse comparative du site osseux par imagerie médicale dans le choix des implants endo-osseux. Thèse d'Odonto-Stomatologie $3^{\mathrm{e}}$ cycle $\mathrm{n}^{\circ} 1$, Dakar, 1998.

9 - Diongue A. Indications des extractions dentaires au Sénégal. Thèse Chirurgie dentaire $2^{\mathrm{e}}$ cycle $\mathrm{n}^{\circ} 4$, Dakar, 2003.

10 - Fugazzotto PA. Implant placement in maxillary first premolar fresh extraction sockets: description of technique and report of preliminary results. J Periodont 2002 ; 73 : 669-74. médecine buccale chirurgie buccale VOL. $15, \mathrm{~N}^{\circ} 4$ 2009 page 207 
11 - Johansson G, Palmquist S, Swenson B. Effects of early placement of a single-tooth implant. A case report. Clin Oral Implant Res $1994 ; 5$ : 48-51.

12 - Krennmair G, Schmidinger S, Waldenberger O. Singletooth replacement with the Frialit 2 system: a retrospective clinical analysis of 146 implants. Int J Oral Maxillofac Implants $2002 ; 17$ : 78-85.

13 - Lazzara RS. Insertion immédiate d'un implant dans un site d'extraction : avantages chirurgical et prothétique. Rev Int Parodont Rest $1989 ; 9$ : 333-43.

14 - Laudenbach P. Radiographie panoramique dentaire et maxillo-faciale (pp. 199-209). CDP, Paris, 1982.

15 - Nemcovsky CE, Artzi Z, Moses O, Gelernter I. Healing of marginal defects at implants placed in fresh extraction sockets or after 4-6 weeks of healing. A comparative study. Clin Oral Impl Res 2002 ; 13 : 410-9.
16 - Parel SM, Triplett RG. Immediate fixture placement: a treatement planning alternative. Int J Oral Maxillofac Implants 1990 ; 5 : 337-45.

17 - Schwartz-Arad D, Gulayev N, Chaushu G. Immediate versus non-immediate implantation for full-arch fixed reconstruction following extraction of all residual teeth: a retrospective comparative study. J Periodontol 2000 ; $71: 923-8$.

18 - Tolman DE, Keller EE. Endosseous implant placement immediately following dental extraction and alveolo plasty: preliminary report with 6 years follow up. Int J Oral Maxillofac Implants 1991 ; 6 : 24-8.

19 - Zarouf H. Implantologie orale : la réhabilitation de l'édente total. Thèse Chirurgie dentaire $2^{\mathrm{e}}$ cycle $\mathrm{n}^{\circ} 16$, Dakar, 2003. médecine 